Psychanalyse et « mensonge organisé » dans le théâtre espagnol des années 1920

« Dites-moi, en cet instant précis, quelle est la limite du mensonge »

\title{
David Coste
}

\section{(2) OpenEdition}

\section{Journals}

Édition électronique

URL : https://journals.openedition.org/cher/297

DOI : $10.4000 /$ cher.297

ISSN : 2803-5992

Éditeur

Presses universitaires de Strasbourg

\section{Édition imprimée}

Date de publication : 19 novembre 2020

Pagination : 15-36

ISBN : 979-10-344-0071-3

ISSN : 1968-035X

\section{Référence électronique}

David Coste, "Psychanalyse et « mensonge organisé » dans le theâtre espagnol des années 1920 ", reCHERches [En ligne], 25

2020, mis en ligne le 01 octobre 2021, consulté le 19 novembre 2021. URL : http://

journals.openedition.org/cher/297 ; DOI : https://doi.org/10.4000/cher.297 


\title{
Psychanalyse et "mensonge organisé» dans le théâtre espagnol des années 1920 "Dites-moi, en cet instant précis, quelle est la limite du mensonge»
}

\author{
DAVID COSTE ${ }^{1}$
}

\section{La traduction des œuvres de Freud en 1922 et l'émergence d'un théâtre «freudien » dans les années 1920}

Le freudisme autoproclamé troisième révolution intellectuelle de l'humanité inflige à l'homme - en posant son hypothèse de l'inconscient -, après les vexations cosmologique copernicienne et biologique darwiniste, la vexation psychologique qui démontre que le «moi n'est pas maître dans sa propre maison »: "Nous devons donc être prêts à admettre en nous, non seulement une seconde conscience, mais aussi une troisième, une quatrième, peut-être une série infinie d'états de consciences, qui nous sont inconnus et qui ne se connaissent pas les uns les autres.» (Freud 2006a: 72). Voilà une construction en poupée gigogne qui laisse d'immenses possibilités au théâtre pour jouer avec la mise en abîme de ces états de consciences multiples et avec les différents niveaux d'énonciation. La question du mensonge, sous ses deux espèces du mensonge à l'autre et du mensonge à soi, suppose d'emblée la dialectique de la conscience et de l'inconscient: le premier impliquant la pleine conscience, le second interrogeant l'inconscient, car comment pourrait-on se mentir à soi-même sans le savoir. Freud invente encore le pré-conscient: voilà qu'il y aurait encore d'autres formes et des états intermédiaires entre ces deux types de mensonge? Que de possibilités pour le théâtre, et le théâtre espagnol des années 1920 en particulier, à la fois sur le plan théorique, esthétique et dramaturgique!

La publication de la traduction espagnole des ouvres de Freud dès 1922 semble aussitôt impulser en Espagne l'émergence d'un théâtre dit "freudien» qui repense la figure traditionnelle du fou théâtral à l'aune des apports de la

1 Professeur agrégé (établissement du secondaire), docteur en études ibériques et latino-américaines. 
psychanalyse, et en particulier de la découverte de l'inconscient. À l'ancienne figure du fou, qui tenait à la fois du bouffon, du bobo et gracioso, et du fou carnavalesque, se substitue le malade mental avec ses traumatismes psychiques. Le facétieux inconscient n'a de cesse de jouer ses tours de passe-passe, libérant sur scène toute une panoplie de (men)songes: mensonges aux autres, mensonges à soi, silences et omissions, travestissements et dénis.

La psychanalyse a introduit une histoire individuelle du traumatisme psychique, a décrit le travail de la triade des systèmes inconscient, conscient, préconscient, du processus de censure et de refoulement, et exploré les symptômes - dans la parole et le corps - qui peuvent en révéler quelque chose. À la médicalisation prônée par la psychiatrie moderne, la psychanalyse propose une nouvelle thérapie basée sur la parole. Il s'agit d'amener le patient à prendre conscience de cette partie - ou vérité - de lui même que l'autre partie, la (pré) conscience, ignore ou plutôt s'arrange pour n'en rien connaître. Cette parole curative, ersatz moderne de la maïeutique socratique, construit l'analyse comme une exploration des formations psychiques et une entreprise démystificatrice des stratégies de la conscience pour ne rien savoir du symptôme, introduisant des concepts qui intéresseront le théâtre: la représentation - consciente et inconsciente, substitutive, refoulée - et les mécanismes de figurabilité dans le rêve, l'identification, la transposition, les formations fantasmatiques, le délire et l'affabulation, le refoulement - «qui ne consiste qu'en ceci: mettre à l'écart et tenir à distance du conscient» et "qui laisse des symptômes derrière lui » (Freud 2006a: 47 et 57 -, le déplacement, la condensation, le déni et le clivage.

Le théâtre freudien espagnol des années 1920 met en scène cette thérapie par la parole, une galerie de personnages affectés par les maladies psychiques les plus variées, mais surtout de névrosés - comme les hystériques de Charcot -, ceux dont s'est principalement occupé Freud, et, bien sûr, la figure du "psychiatropsychanalyste» tantôt présenté comme imbu de sa nouvelle science, l'étalant avec arrogance, tantôt comme un savant fou, utopiste aspirant à réformer l'humanité. Ainsi le théâtre, art qui met au premier plan l'homme et sa relation à l'autre, la parole et le dédoublement sous toutes les espèces et à tous les niveaux - de l'être et du paraître, du travestissement, du double, des incarnations et désincarnations, de l'acteur et du personnage, de la double énonciation etc. ne pouvait que trouver une matière des plus riches dans ces découvertes de la psychanalyse, cette "psychologie des profondeurs» comme elle a été désignée à l'époque. Cette nouvelle science n'offrait-elle pas au théâtre des pistes et des outils pour explorer les formes les plus diverses de «théâtres du je» - théâtre symboliste et expressionniste - et de jeux de mots et de gestes, de mots et de «figurations plastiques $»^{2}-$ dans sa recherche d'un "théâtre total » faisant appel à

2 «Il est très remarquable de voir comment le travail du rêve s'attache peu aux représentations de mots; il est à chaque instant prêt à échanger les mots les uns pour les autres jusqu'à ce qu'il trouve l'expression qui offre à la figuration plastique le plus de commodité.» (Freud 2006a:133). 
tous les arts? Ne lui ouvrait-elle pas de nouvelles perspectives pour démultiplier et enrichir dramaturgiquement les jeux de faux-semblants et d'apparences, de rêve - du rêve éveillé à la "confusion hallucinatoire de l'amentia ${ }^{3}$ » (Freud 2006a : 135) - et de réalité - «l'épreuve de réalité»-, de (men)songes et de vérités?

À la lumière de la psychanalyse, le théâtre revisite la place du corps, du réel et du phantasme, joue de l'ambivalence, explore de nouveaux dispositifs de redoublement ${ }^{4}$, de retournement, de renversement, d'inversion ou de brouillage des rapports entre regardants et regardés, regarder et être regardé, activité et passivité, entre l'acteur et son double, entre l'être et le paraître, le vrai et le faux, la réalité et le rêve - avec son travail de "condensation", "déplacement" et "dramatisation» et ses interprétations multiples -, le vraissemblable et l'irrationnel. D'autant plus que cette nouvelle "psychologie des profondeurs» investit le champ sémantique et métaphorique du théâtre.

Si certaines formes théâtrales ont plutôt fait intervenir la psychanalyse pour la tourner en dérision, ne la mettant en scène que pour mieux la remettre en question, ou plus prosaïquement parce qu'elle se prête à la création de comiques de mots et de situations, d'autre semblent bien constituer une reconnaissance de l'importance de la psychanalyse et de la voie qu'elle ouvre pour des stratégies et esthétiques dramaturgiques qui visent à rénover, "rethéâtraliser» - lieu commun des avant-gardes théâtrales - un théâtre espagnol en crise - «la crise du théâtre» est un autre leitmotiv de l'époque -. Si le théâtre des années 1920 est en quête d'un renouveau esthétique, c'est aussi dans un sens politique, dans l'Espagne de ces années où la fracture de la société espagnole qui est bien entamée déjà, débouchera sur la guerre civile espagnole de 1936.

Cette science du psychisme de l'homme invite ainsi le théâtre à revisiter la question existentielle, politique et métathéâtrale de la dialectique véritémensonge; à réactualiser, en les désacralisant et en les réélaborant sous l'angle psychanalytique, les allégories caldéroniennes du "grand théâtre du monde » et des habits du fou ${ }^{5}$.

3 «L'amentia est la réaction à une perte que la réalité affirme mais que le moi doit dénier, parce qu'insupportable.» (Freud 2006a: 141).

4 " [...] nous savons ainsi que le rêve est absolument égoïste et que la personne qui joue le rôle principal dans les scènes, s'avèrera toujours être la personne propre.» (Freud 2006a: 125).

5 Calderón de la Barca: «Si je revêts l'habit du fou, ce n'est pas parce que je le suis, mais parce que la prudence me guide lorsque je suis en public / car il n'y aurait pas fou plus incurable que celui qui dirait tout ce qu'il pense." (cette traduction libre des vers de Calderón est de nous).

«Andar de loco vestido / no es porque a solas lo soy, /sino que en publico estoy / a la prudencia rendido, / pues ningún loco se hallará, / que más incurable fuera, / si ejecutara, y dijera / un hombre cuanto pensara: / y así, lo parecen pocos, / siéndolo cuantos encuentro, / porque visto hacia dentro, / todos somos locos, / los unos, y los otros.». (La cena de Baltasar, Calderón de la Barca 1717: 206). 
L'analyse portera sur deux pièces représentatives de cette veine théâtrale des années 1920 en Espagne: Sinrazón. Juguete trágico en tres actos (1928) d'Ignacio Sánchez Mejías (1891-1934) et Tararí. Farsa cómica en dos actos y un epílogo (1929) de Valentín Andrés Álvarez (1891-1982).

Sinrazón raconte la refondation d'un asile dirigé par le psychanalyste en herbe Ballina sous l'impulsion de son riche patient Don Manuel. L'Asile, transformé en un palais, le palais de la reine Béatrice, permet à ses "hôtes" de vivre leurs obsessions et rêves en leur donnant les moyens matériels d'être ce qu'ils croient être. Mais la stratégie curative du Palais de Béatrice tourne au drame lorsque le docteur Ballina réalise que ce n'est pas le moyen d'obtenir la guérison et applique sa méthode de la cure de la parole pour dénouer le phantasme et faire émerger le noyau de résistance et la vérité. Le troisième acte met en scène les divergences d'opinion, chaque fois plus marquées, entre Ballina et Don Manuel, préfigurant la catastrophe finale:

BALlina.- [...] Notre orientation actuelle n'est pas bonne, car c'est un véritable non-sens que d'encourager, comme nous le faisons, la folie de ces malheureux. C'est précisément le contraire que nous devons faire. [...] Il faut opérer sur la conscience, faire en sorte que le malade découvre l'origine de sa maladie, qui est presque toujours dans l'ignorance de celle-ci; [...] renforcer les digues de sa conscience $[\ldots]^{6}$.

D. Manuel. - [...] Je commence à douter de votre raison. Dénuder les gens de l'intérieur? Leur révéler ce que l'on pense d'eux? Leur dire la vérité, la vérité toute nue? Non, docteur, d'aucune manière. Ici on ne dit la vérité à personne, absolument à personne $[\ldots]^{7}$.

L'expérience psychanalytique tourne court. Don Manuel finit par étrangler le représentant de la psychanalyse et le rideau tombe sur un asile qui sombre de plus belle dans la folie, puisque les fous-soldats gardent désormais la forteresse du «mensonge organisé», terme que l'un des personnages emploie pour désigner la folie. Le spectateur aura beaucoup ri, il aura été complice de la supercherie; il sera entré dans la folie douce de personnages qui ne sont que des silhouettes se découpant sur le fond onirique de ce conte qu'introduit une citation, en voix off, de Nietzsche: "Ce que nous vivons en rêve, si nous le vivons fréquemment, appartient, au bout du compte, à la totalité de notre âme, comme n'importe quelle autre expérience vécue. [...] en plein jour, même lorsque notre esprit éveillé est le plus serein, nous sommes conduits par la main par l'habitude

6 «BALlinA.- [...] Nuestra orientación de hoy no es buena, porque es un verdadero disparate fomentar, como hacemos, la locura de estos desgraciados. Es precisamente todo lo contrario el plan a realizar. [...] hay que operar sobre la conciencia, descubrir al enfermo el origen de su enfermedad, que casi siempre es ignorado por ellos; [...] reforzar los diques de su conciencia [...]» (Sinrazón, p. 48-50)

7 «D. Manuel. - [...] Empiezo a dudar de su razón. ¿Desnudar a la gente por dentro? ¿Descubrirles lo que pensamos de ellos? ¿Decirles la verdad, la verdad absolutamente desnuda? » (Sinrazón, p. 50) 
de notre rêve $^{8}$.» Le spectateur peut-il vraiment ressentir le dénouement de Sinrazón comme tragique? N'est-ce pas finalement rien de plus qu'un jeu qui a mal tourné... dans le rêve... Une double utopie - le (men)songe du palais de don Manuel et la vérité à tout prix de la thérapie du docteur Ballina - qui s'est écrasée contre la réalité? Ce que pointe le sous-titre générique de Sinrazón: «jouet tragique» («juguete trágico»).

Tararí est par contre qualifiée de «farce comique». La pièce met en scène le soulèvement des fous qui prennent le pouvoir dans l'asile et installent leur ordre, celui de la folie. Mais, la nouvelle organisation de l'asile est à l'image de celle qui la précédait: la folie s'impose par la force; les nouveaux maîtres du jeu ont recours aux mêmes procédés qu'utilisaient leurs prédécesseurs au nom de la raison, c'est-à-dire l'enfermement, la camisole chimique et physique. Nous assistons ainsi à une inversion des rôles - lieu commun du théâtre, mais aussi motif principal de la fête des fous - qui semble prendre fin lorsque les forces de l'ordre investissent le huis clos de l'asile. Cependant, chacun des internés a si bien assimilé son nouveau rôle, ils sont si criants de vérité dans leurs nouveaux habits sociaux, que les forces de l'ordre s'y laissent prendre. Nous assistons ainsi à un deuxième retournement final, inattendu, puisque ceux qui devaient être libérés sont pris pour les fous. Dénouement plein de paradoxe. Il pourrait être lu comme subversif, sorte de justice théâtrale, il ne l'est pas vraiment au bout du compte puisque le nouvel ordre installé est le calque exact du précédent. Il est plutôt le constat cynique, applicable à l'ensemble de la société, qu'il vaut mieux, pour éviter la camisole de force, se taire que s'agiter.

On perçoit, avant même d'entrer dans les textes, la complexité et la multiplicité des niveaux de lecture - asile, société, condition humaine -, des jeux de masques et contre-masques, de trompe-l'œil, de vrai et de faux, de vérités et de mensonges, d'illusions et de faux-semblants. Loin d'avoir affaire à un théâtre de la vérité révélée, nous avons un dispositif pyramidal de vrais-faux et de faux-vrais. Toutes les formes de contrefaçons de la vérité trouvent une place dans ces comédies de la confusion et de l'inversion des identités: mensonge à soi et mensonge à l'autre, dans toutes ses gradations - cyniques et perverses ou ludiques et joyeuses -; illusion, hallucination et mise en scène d'un rêve devenu réalité collective des fous, c'est à dire la prise de pouvoir dans Tararí; supercherie médicale du médecin porte-parole d'une vérité et d'une science qu'il manie à sa guise selon ses intérêts.

8 «Voz.- (De Nietzsche.) Aquello que vivimos en sueño, siempre que lo vivimos con frecuencia, pertenece, al fin y al cabo, a la totalidad de nuestra alma, como cualquier cosa realmente vivida. [...] en pleno día, incluso en los más serenos instantes de nuestro espíritu despierto, somos llevados un poco de la mano por los hábitos de nuestro sueño.» (Sinrazón, p. 23-24) 
Quand je est un autre: inversion, faux-semblants, délire de personnalité et croyance

Les deux pièces nous situent dans un asile, monde forclos non pas tant pour protéger malgré eux les malades, que pour épargner à la société la contagion de ces délires - point d'entrée de la critique sociale de ces pièces. Tout au moins est-ce l'une des interprétations à laquelle nous invite leurs auteurs. Elles jouent métathéâtralement, dans l'élaboration du délire de personnalité, du double huisclos, celui fictionnel que construisent les murs cloisonnant de l'asile - la fiction de la folie -, et ceux, réels, de l'espace scénique et du lieu théâtral, tout aussi cloisonnés, d'une part, par le quatrième mur, et, d'autre part, ensemble, huis clos séparé du monde extérieur. Dispositif double qui confronte et sépare un intérieur et un extérieur.

La frontière des murs de l'asile, au départ établie par la société excluante, est renforcée dans les deux pièces par les internés eux-mêmesqui ne laissent plus personne sortir. L'asile encore ouvert sur l'extérieur au début de la pièce, se referme hermétiquement dans le dénouement, renforçant son système de contrôle - par des patients-soldats dans Sinrazón, par un commissaire et les forces de l'ordre dans Tararí. Qui entre dans l'asile, n'en ressort pas.

L'hermétisme doit être total dans Sinrazón pour ne pas risquer de compromettre l'expérience imaginée par don Manuel: rien ne doit perturber le délire de personnalité des patients, il convient de leur offrir les conditions les plus favorables à l'épanouissement des phantasmes. La sortie de l'asile n'est pas envisagée, les murs doivent préserver ces «mensonges organisés » - expression que l'un des personnages utilise pour définir la folie - contre l'irruption de la réalité extérieure. L’objectif du docteur Ballina est par contre de les faire sortir, de les réintégrer à la société. À la fin de la pièce, avec la mort de Ballina, l'asile se referme sur lui-même, à double tour; des «soldats» se positionnent le long du mur-forteresse. Dans Tararí, la séparation stricte avec l'extérieur est maintenue par les internés eux-mêmes. Ils n'aspirent pas à sortir, juste à imposer leur ordre - un monde à l'envers - à l'intérieur de l'asile. Ils verrouillent toute possibilité de sortie. Si on peut entrer dans l'asile, pas question en effet d'en ressortir. Tout individu sain qui s'y aventure est aussitôt accueilli comme malade par les internés qui ont pris le pouvoir. C'est ce qui arrive au Visiteur, riche commerçant, et au Notaire.

Dans les deux œuvres, nous avons la figure d'un fou enfermé dans un asile dont la folie est de se croire un autre que ce qu'il est. Fort habile dans l'élaboration de son délire et jamais à court d'arguments, il est sa propre dupe tout en dupant son monde, à moins qu'il ne dupe les autres, et, s'y laissant prendre, ne se dupe lui-même. Totalement identifié à sa nouvelle personnalité, y croit-il sans autre distance, se prenant vraiment pour cet autre, ou, comme l'acteur sommé de jouer son rôle avec "sincérité» et "authenticité», incarne-t-il seulement un personnage, s'appliquant à lui donner toute la force de la vraissemblance? Belle 
manière métathéâtrale de jouer avec les notions de "personnage» et de «rôle»; belle manière encore de jouer - se jouer $\mathrm{du}$ - avec le spectateur.

Dans Sinrazón, nous avons le délire de celui qui se croit un évêque et qui remodèle les lieux et ceux avec qui il entre en interaction pour mieux affirmer et consolider sa nouvelle identité. Il met la logique de l'argumentation et le raisonnement cohérent au service de sa fiction. Il réinterprète ainsi la réalité à la lumière de cette autre réalité, sa réalité. Fort documenté sur ce qu'est un évêché et son fonctionnement et sur certaines questions de théologie, pièce par pièce il construit son château de cartes:

Madame sait que je ne peux pas être cardinal tant que l'on n'a pas reconnu mon statut d'évêque. On ne m'a toujours pas remis mes papiers. Des manœuvres politiques de Rome. Et des Augustins. Le concept théologique de la grâce nous sépare $[\ldots] .{ }^{9}$

Ici, pas d'intentionnalité malveillante, pas de désir de tromper l'autre, pas de mensonge. Le délire du fou psychotique certes crée une fiction, qui, si elle est telle pour les autres, n'en demeure pas moins la réalité pour lui. S’il ne s'agit pas pour lui de tromper l'autre, il a besoin cependant de l'autre pour construire sa réalité et donc lui donner toute sa consistance. Il en est de même d'un autre personnage de la pièce, celle qui se croit reine:

Sceur Victoire.- Ne sois pas bête. Tends ta main à ce monsieur, allez. (Elle essaye de la prendre par la main).

REINE (avec défi).- Ma main, non Sœur Victoire, on ne touche pas à ma main. Ma main est sacrée, sacrée. Seul celui qui a la grâce peut la baiser. C’est ainsi que le souhaite le roi. (Baissant la voix, rougissant, assaillie par les mêmes désirs.) Le roi. C'est ainsi que le souhaite le roi, le roi. (Elle se cache le visage entre les mains avec malice.) Le roi. ${ }^{10}$

La parole insistante, redondante et péremptoire de la «reine» interdit toute contestation. On pressent que tout démenti pourrait non pas tant réduire à néant la fiction que faire exploser psychiquement celle qui est pour elle-même, et donc pour les autres, une reine qui attend son roi.

Cette situation est l'occasion pour une réécriture du motif de la cécitéclairvoyance du fou, largement exploité dans la littérature et le théâtre en particulier. Nous rappellerons que ce trait spécifique de la clairvoyance du fou dans sa folie est l'une des idées tout aussi amplement développée dans l'Occident chrétien: la folie indique la main et l'œuvre de Dieu. Le dément voit ce que

9 «La señora sabe que yo no puedo ser cardenal mientras no se reconozca mi obispado. Todavía no me entregan mis documentos. Maniobras políticas de Roma. Y de los Agustinos. El concepto teológico de la gracia nos separa...» (Sánchez Mejías 2010 : 55).

10 «Sor Victoria.- No seas tonta. Dale la mano a este señor, vamos. (Intenta cogerla de la mano.)

Reina.- (Bravamente.) Mi mano, no, Sor Victoria, mi mano no se toca. Mi mano es sagrada, sagrada. Sólo la besa el que está en gracia. Así lo quiere el rey. (Bajando la voz, ruborizada, acometida de los mismos deseos.) El Rey. Así lo quiere el Rey, el Rey. (Esconde la cara entre sus manos maliciosamente.) El Rey.» (Sánchez Mejías 2010: 39). 
d'autres ne peuvent voir; il peut prédire l'avenir. Il est perçu comme un «poète clairvoyant» (Heers 1983: 142). La pièce de Sánchez Mejías subvertit ce modèle et se débarrasse de la main divine: ses fous ont perdu en acuité.

Certes, la reine porte des lunettes qui sont censées la doter de cette clairvoyance, mais il ne s'agit que d'un "face-à-main en toc» ("falsos impertinentes»), loin de lui donner ce don de Dieu. La clairvoyance supposée du fou ne serait déjà plus ici un don de Dieu puisque «la reine» a besoin d'un artefact, les lunettes, qui, de plus, se révèlent ne pas en être. Mais les lunettes permettent, symboliquement, à celle qui se prend pour une reine de voir la réalité en cohérence avec, non pas ce qu'elle souhaite ou croit être, mais bien avec ce qu'elle est en ce moment précis. Ainsi les lunettes, outils du bien voir, du mieux voir, deviennent au contraire l'adjuvant de la cécité du fou, de la fiction et de l'illusion, artefact nécessaire à la construction de ce mensonge à soi filé qu'est le délire de personnalité. Mais l'évêque et la reine de Sinrazón croient-ils vraiment à leur délire ou ne jouent-ils pas seulement à y croire? N'y a t-il aucune faille dans ce mentir à soi même? Ils construisent une fiction qui, pour se doter de tous les contours et la force de la réalité, nécessite l'adhésion des interlocuteurs. La recherche insistante d'une telle adhésion ne laisse-t-elle pas encore se glisser le doute? Ne faut-il pas en savoir malgré tout quelque chose? Ne s'agirait-il pas dans cette recherche d'une adhésion à tout prix d' "un vouloir faire croire» plutôt que d'un croire. Derrida rappelle que «le mensonge est un vouloir faire croire" (Derrida 2005: 15). Sommes-nous dans cette configuration avec les personnages de Sinrazón? Tous leurs efforts ne relèveraient-ils pas plutôt d'une affirmation entêtée de leur condition comme pour mieux se convaincre qu'ils sont ce qu'ils ne sont pas, comme pour mieux y croire et y faire croire? Dans ce cas, il y aurait bien intention de construire une fiction pour soi et pour les autres, de se mentir à soi et aux autres. Il y aurait dès lors conscience de se mentir. La pièce joue bien sûr de tous les ressorts dramaturgiques qu'offrent ces ambiguïtés pour construire sa fable et le suspens. Cependant la question, finalement, n'est pas une question de conscience ou d'inconscient, de vérité ou de mensonge, mais de croyance. Les analyses du philosophe Nicolas Grimaldi nous éclairent sur ce point:

La croyance n'a pas besoin de lien avec la réalité. Plus elle est loin de la réalité, plus elle est solide. Sa solidité est à la mesure de son éloignement du réel. Moins elle m'offre la possibilité du démenti, plus elle est solide. C'est ici que l'on peut parler en un sens de folie, c'est-à-dire d'un système qui, en aucun cas, ne s'expose à la possibilité d'être réfuté par la réalité. (Grimaldi 2011: 103)

Les personnages délirants de Sinrazón réinterprètent chacun des signes dans le sens de leur délire. Les sens en alerte, ils sont à l'affût du moindre signe qui les confirme dans leur réalité. Ils sont occupés «à imaginer une signification » à chacun de ces signes:

Sans doute, toute perception nous impose-t-elle une réalité. Mais ce n'est la réalité que d'un signe ou d'un ensemble de signes. À ces signes il faut imaginer une signification. Et c'est la croyance plus ou moins bien fondée à cette signification 
qui détermine notre perception. Si furtivement ou provisoirement que ce soit, percevoir c'est croire. (Grimaldi 2009: 78)

Dans Sinrazón, la reine imagine une signification qui va dans le sens de son délire - elle est la reine - à chacun des bruits qui lui parviennent du hors-scène:

(Un coup de clairon interrompt les dernières paroles de Don Manolito.)

REINE (Avec extase).- Le roi!

Don Manuel. - La relève de votre garde.

REINE (capricieuse et insistante). - Le roi! Le roi! Le roi!11

\section{La fabrique d'un délire collectif ou le monde à l'envers}

Le mensonge à soi, dans les délires de la personnalité mis en scène dans ces deux pièces, ne peut s'imposer ni se maintenir que s'il est relayé par les autres. Pour qu'il existe, il faut que les autres croient - ou feignent de croire - à cette réalité comme réalité et non comme mensonge, qu'ils aient - ou feignent d'avoir - la même perception des signes extérieurs, qu'ils donnent la même signification à ces signes. Le huis-clos est la première condition pour que puisse se produire ce délire collectif, c'est-à-dire la mise en place de cette nouvelle réalité. La deuxième condition est la règle suprêmede ce jeu que chacun est sommé de ne pas enfreindre: on joue le jeu, on ne contredit pas, on ne dément pas. Ce mensonge collectif présente des caractéristiques différentes dans chacune des deux pièces. Dans Sinrazón, mise en scène du délire d'amour de la reine, les soignants s'impliquent totalement dans ce délire, y participent activement. Dans Tararí, où les fous ont pris le pouvoir, nous avons affaire à un contre-délire subversif. Mais, dans les deux cas, finalement, l'institution asile est l'otage du bon vouloir des «fous», et le délire collectif est celui d'un monde à l'envers.

Dans Sinrazón, à la fin du premier acte, le médecin expérimente la technique du psychodrame. C'est le point de départ du mensonge collectif. À l'origine, le psychodrame, technique psychiatrique, consiste dans la mise en scène de l'illusion du malade à des fins cliniques curatives, pour libérer le malade de ses obsessions, traumatismes ou phantasmes. Ce procédé, déjà en soi théâtral, est appliqué dans Sinrazón, comme il l’a déjà été en 1922 par Pirandello dans Henri IV - pièce dont le thème est aussi la folie - où le protagoniste, depuis sa chute de cheval vingt ans auparavant, se prend pour l'empereur Henri IV du Saint Empire. Tout son entourage, comme dans Sinrazón, joue le jeu dans le rôle de sa cour. Le modèle pirandélien est on ne peut plus prégnant dans la pièce de Sanchez Mejías. Il s'agit donc d'entrer dans l'illusion du patient pour le soigner

11 "(Un toque de clarín corta las últimas palabras de don Manolito.)

REINA.- (Con éxtasis.) ¡El Rey!

Don MANUEL. - El relevo de vuestra guardia.

ReInA._(Caprichosa einsistente.)¡El Rey! ¡El Rey! ¡El Rey!»(Sánchez Mejías 2010 :55). 
«de l'intérieur». Ainsi l'exposent de concert Ballina et Don Manuel, son riche patient et mentor, dès le début de la pièce:

BALLinA. - Nous allons créer une légende et lui donner vie. Une reine, prisonnière de l'amour, attend dans son palais le roi pour se marier. Et le roi n'arrive jamais [...]. Don Manuel, c'est ce que nous projetons, n'est-ce pas?

Don Manuel. - Exactement. Nous allons créer une légende et lui donner vie. ${ }^{12}$

La mise en place du psychodrame s'apparente à une mise en scène théâtrale. Pour soigner celle qui se prend pour une reine, et apporter du bonheur aux "pauvres fous», à «ces malheureux» (Sinrazón: 29, 31, 32), l'espace de l'asile est transformé entièrement en un palais, le palais de la reine Béatrice, avec ses décors et ses figurants: «Ballina. - Non. Fi de l'asile. Un palais où une reine aura sa cour. N'est-ce pas mon cher Don Manuel? Fi des fous. On n'entendra plus jamais cet horrible mot dans notre palais ${ }^{13}$." Une nouvelle folie à deux se prépare? En réalité, comme le révèleront la fin de l'acte II et l'acte III, Ballina et don Manuel n'ont ni les mêmes objectifs, ni la même vision de la nouvelle science psychanalytique. Tous les occupants, médecins, infirmiers et internés, sans exception, sont donc mis à contribution pour incarner les rôles nécessaires à la mise en place de la fiction de l'asile-palais de la reine Béatrice. Le scénario imaginé dans l'acte I est mis en application dans l'acte II. Les deux expérimentateurs prévoient les moindres détails du travestissement, les signes à mettre en place pour qu'ils soient interprétés dans le sens voulu. En un mot, ils vont offrir à la malade des signes et une fiction qui la consolideront dans son délire. Non seulement le médecin joue le jeu du délire de la reine, mais il entre dans son jeu, va bien au-delà puisqu'il construit la fiction et alimente sa folie, et finalement s'y laisse prendre. Le temps de cette mise en scène, la fiction doit rester la réalité: aucun signe, aucune intervention ni aucun démenti ne doivent venir briser l'illusion. Cependant, ce que révèle déjà l'acte II, c'est que pour le docteur Ballina l'objectif est de "guérir" la patiente et lui permettre de quitter l'asile - et donc de défaire l'illusion -, alors que pour don Manuel l'objectif est d'offrir un cadre de vie idyllique, utopique, où chacun puisse continuer à rêver «la folie est un rêve filé » (Sinrazón, p. 48, p. 60) - et ainsi contribuer au bonheur des internés. Si le docteur Ballina favorise le délire de la reine, c'est pour lui permettre ensuite de mieux s'en déprendre, comme un acteur retire ses habits de scène à la fin de la représentation, comme le spectateur revient à la réalité quand le rideau tombe. L'un vise donc la guérison et la sortie de l'asile, l'autre l'amélioration des conditions de vie des fous dans l'asile et la sauvegarde de leurs

12 «BALLinA. - Vamos a crear una leyenda y a darle vida. Una reina, prisionera de amor

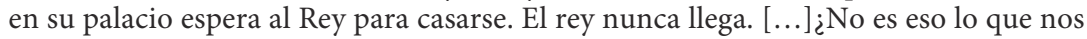
proponemos, don Manuel?

D. Manuel. - Exactamente. Vamos a crear una leyenda y darle vida.» (Sinrazón, p. 31-32).

13 «Ballina. - No. Nada de manicomio. Un palacio donde una reina tenga su corte, ¿verdad, don Manolito? Nada de locos. La horrible palabra no se oirá nunca en nuestro palacio.» (Sinrazón, p. 31). 
délires. L'un voit le salut dans la réintégration dans le monde extérieur, le second dans la mise à distance de ce monde extérieur. La comédie psychanalytique invite à la satire sociale. D'ailleurs les délires des fous nous mènent sur ce terrain: les fous-soldats et gardes de la reine, la reine et sa cour, l'évêque, le commerçant et spéculateur.

Dans Tararí, la mise en place du mensonge collectif se fait à partir d'une autre tradition - caractérisée aussi par des manifestations (para-)théâtrales -, celle du renversement carnavalesque des rôles propres à la fête des fous. Les internés prennent le pouvoir et usurpent les identités de ceux qui les contrôlaient jusque-là. Dans leurs nouveaux rôles, qu'ils s'appliquent à représenter à la lettre, ils assurent à leur tour l'ordre dans ce nouvel équilibre - inversé - des pouvoirs en usant des mêmes instruments de répression: la camisole de force. Que les anciens maîtres des lieux protestent, les voilà aussitôt emprisonnés dans l'une de ces camisoles. Chacun revêt ainsi le costume de scène du nouveau rôle. Comme dans Le Grand théâtre du monde de Calderón de la Barca, où le personnage Monde distribue aux acteurs le texte, les objets et symboles de leurs rôles, au nom de Dieu, ici on assiste à une redistribution des attributs pour une nouvelle répartition des rôles. L'habit fait bien le moine.

Même si dans les deux pièces, au bout du compte, on se demande qui est le plus fou, nous avons deux types différents de retournement, mais un même dénouement: dans les deux cas, l'asile se referme sur lui-même plus que jamais: plus personne n'en sortira. Dans Sinrazón, les malades, clairement montrés dans leur délire, croient vraiment ce qu'ils ne sont pas. Le psychanalyste finit par se laisser prendre dans les filets d'un contre-transfert mal géré. La pièce s'achève sur l'échec de l'expérience psychanalytique du docteur Ballina qui devait permettre aux patients de quitter l'asile. Dans Tararí, nous assistons à la prise de pouvoir par des malades qui contrôlent désormais l'asile.

Dans les deux paraboles théâtrales, de l'expérience clinique dans Sinrazón, de l'inversion des rôles dans Tararí, le délire collectif, orchestré par le médecin dans un premier cas, par les malades dans le deuxième, n'est-il pas surtout à interpréter comme un microcosme à l'image du macrocosme extérieur, du délire social et politique d'une société malade, celle de l'Espagne des années 1920 ? Les deux «mensonges organisés» mis en scène dans ces pièces ne nous parlent-ils pas davantage du disfonctionnement général d'une société minée par les corruptions, les mensonges et les faux-semblants, que de ces "pauvres fous"? L'échec de l'expérience scientifique de Don Manuel dans Sinrazón et l'ordre renversé remplacé par un ordre qui reproduit la même structure répressive dans Tararí en disent long sur le découragement et le pessimisme quant aux solutions pour relever une Espagne au bord du chaos. 


\section{La dialectique facétieuse et retorse du mensonge ou comment rendre I'autre fou}

Les deux pièces invitent aussi à une réflexion plus générale sur le mensonge et la folie comme «mensonge organisé» (Sinrazón, p. 25). Elles mettent en scène une histoire - récurrente dans la comédie et la farce - de trompeurs trompés, illustrant ce que disent beaucoup de proverbes dans leur sagesse populaire: "Les plus trompeurs ne sont pas les moins trompés», "Plus d'un trompeur se prend au piège qu'il dressait à autrui. " Elles décortiquent les intentions et les stratégies tortueuses du mensonge, mensonge à l'autre et mensonge à soi. Elles explorent par le rire les dimensions poétiques, sociales, politiques et morales du mensonge, du mensonge le plus pervers au mensonge blanc. Elles multiplient les jeux de brouillages. Par exemple, à la fin du premier acte (p. 38) de Tararí, un Visiteur frappe à la porte de l'asile et demande à rencontrer le directeur de l'asile. Don Paco, l'un des meneurs des fous qui ont pris le pouvoir, se présente à lui dans ce rôle, mais sous son vrai nom, tout en précisant: "Lorsque on entre dans cette maison on ne vous dit jamais la vérité.» (p. 39), remarque bien sûr qui ne peut être comprise par le Visiteur, mais que ce dernier prend avec sérieux au pied de la lettre. S'en suit un dialogue portant sur le mensonge, d'autant plus savoureux pour le spectateur en position de sur-savoir qu'il a lieu au moment même où le Visiteur est en train d'être berné par le faux directeur:

Visiteur.- Monsieur le directeur de l'établissement?

Don PACo. - En personne.

Visiteur. - Donc c'est vous le...

Don Paco.- (L'interrompant.) Don Paco...

Visiteur.- (Surpris.) Don Paco?... (Il sort une lettre.)

Don Paco._- Oui, don Paco...

Visiteur. - Comment se fait-il alors qu'on m'ait dit que vous vous appelez...?

Don PACo.- On vous a berné. Mais cela n'a rien d'exceptionnel. Quand on vient dans cette maison on ne vous dit jamais la vérité. Tous arrivent ici bernés.

Visiteur.- (Reculant.) Monsieur, vous me confondez...

Don Paco.- Moi? Mais je me suis limité à vous dire que vous êtes arrivé ici berné, comme tous les autres. Est-ce de ma faute s'il en va ainsi?

Visiteur.- Oui, mais vous comprendrez que ce n'est pas non plus de ma faute si un ami s'est moqué de moi, m'a dit qu'il connaissait très bien le Directeur de cette maison et m'a adressé à vous avec cette lettre de sa part. N'importe qui peut être victime du mensonge d'un scélérat.

Don PACo.- Ne lui faites pas non plus porter la faute. C'est un mensonge si blanc. ${ }^{14}$

14 «Visitante. - ¿El señor Director de este establecimiento?

Don PACO. - Servidor.

Visitante.- De manera que usted es...

Don Paco.- (Atajándolo.) Don Paco... 
Le pauvre Visiteur venu se renseigner pour interner son frère malade et de surcroît philosophe, qui ose à peine prononcer le mot tabou, "fou», se trouve piégé par la dialectique sans fond du facétieux et retors Don Paco qui retourne la situation en faisant mine de comprendre que l'homme vient en réalité se renseigner pour lui-même: "Vous avez réveillé à ce point mon intérêt que je désire m'occuper de vous plutôt que de votre frère", (Tararí, p. 40), "Alors comme ça vous n'êtes pas venu pour vous mais pour votre frère! Eh bien! On vous a bien doré la pilule!» (p. 42). Les autres fous se joignent à Don Paco pour jouer un mauvais tour à ce visiteur, riche commerçant de son état et si plein de préjugés sur les fous. Le Visiteur ne sait plus où il en est, dans une piètre imitation du Sigismond de La vie est un songe, il s'exclame: "mon dieu, ce que j'ai sous les yeux, est-ce la réalité ou l'illusion d'un rêve?» (p.43). Les fous ne perdent pas l'occasion de se lancer ironiquement dans un nouveau développement du "problème métaphysique de la réalité» (p. 44). Chaque précision que le Visiteur apporte pour les détromper - il n'est pas le frère philosophe "fou", mais le frère commerçant sain d'esprit - est retournée comme un gant: "Ne vous inquiétez pas. Vous serez très bien ici. Les malades sont très bien traités, nous avons un magnifique jardin où vous pourrez vous promener avec d'autres compagnons.» (p. 45). Le Visiteur, dans la réplique de clôture de l'acte, finit par accepter sa nouvelle identité, il est bien le frère philosophe fou: il se met alors à dire toute la vérité sur celui qu'il n'est plus, c'est-à-dire le frère commerçant: «l'épicier est un scélérat. Un scélérat. Et je sais de quoi je parle!» Et trouve même des avantages à la situation: "donc, la femme de mon frère, qui m'a toujours beaucoup plu... est ma femme. Eh, qu'on me l'amène, oui, eh, qu'on me l'amène.» (p. 47). La supercherie, parodie de cure par la parole, aura eu les effets d'une cure par la parole, puisque le Visiteur aura énoncé la vérité sur lui-même. Mais cette vérité est révélée au prix d'un «mensonge organisé», sa nouvelle folie de se prendre pour son frère, suscitée par manipulation. Le résultat est un Visiteur désorienté qui ne sait plus qui il est. À la fin de la pièce, il participera "sincèrement» à la confusion générale quand le commissaire et les forces de l'ordre sont incapables

\footnotetext{
Visitante.- (Extrañado.) ¿Don Paco? ... (Saca una carta.)

Don Paco.- Sí, señor, don Paco...

Visitante.- ¿Cómo me han dicho a mí entonces que usted se llamaba?...

Don PaCo. - Le han engañado. Pero no tiene nada de particular. Cuando uno viene a esta casa nunca le dicen la verdad. Todos vienen aquí engañados.

Visitante.- (Retirándose.) Usted, señor, me confunde...

Don Paco.- ¿Yo? Pero si sólo le he dicho que ha venido usted aquí engañado como vienen todos...¿¿Qué culpa tengo yo de que sean así las cosas?

Visitante.- Sí, pero comprenderá que tampoco tengo yo culpa alguna de que un amigo se haya burlado de mí diciéndome que conocía mucho al Director de esta casa y me haya hecho venir con esta carta para usted. Cualquiera puede ser víctima de la mentira de un sinvergüenza.

Don PAco.- No le culpe demasiado a él tampoco. ¡Es una mentira tan piadosa!» (Tararí, p. 38-39).
} 
de discerner qui sont les fous et qui sont les membres institutionnels de l'asile qu'ils sont venus soutenir :

Commissaire. - Mais lesquels sont les fous?

Don Paco.- Vous ne les reconnaissez pas à leurs cris d'énergumènes et leurs gestes désordonnés?

Commissaire.-Ceux-ci? (Il signale les sains d'esprit).

Don Paco.- Oui, oui, c'est ça... et il faut leur passer la camisole sur le champ.

Les SAINS D'ESPRIT.- (À grands cris.) Ne l'écoutez pas, Monsieur le Commissaire. Les fous ce sont ceux-là... Nous, nous sommes les sains d'esprit, oui, Monsieur, les sains d'esprit.

Don Paco.- Comme vous le voyez, nous, nous sommes les fous pacifiques et eux les sains d'esprit à lier.

$[\ldots]$

Visiteur.- Moi, monsieur, je suis un pauvre cinglé. Je suis ici au milieu de cette confusion parce que... franchement, je ne sais plus quel est mon camp et je souhaiterais que vous me le disiez.

Commissaire.- Que je vous le dise? (Regardant chacun des deux groupes.) Mon cher ami, je n'ai pas une telle acuité. (Il reste, un instant, pensif.) Mais, bon, voyons, n'y avait-il pas un directeur ? ${ }^{15}$.

Comme nous le voyons, s'appuyant sur une réalité sociale, les préjugés et les stéréotypes des fous, Don Paco retourne la situation à l'avantage des fous, pour rendre fou le commissaire et renforcer la confusion générale. La folie est si relative, montre la pièce.

Dans Sinrazón, le psychiatre Ballina se propose d'appliquer une nouvelle technique, la cure analytique. Il va la mener d'une façon très personnelle. La pièce, loin d'encenser la nouvelle science, est un véritable réquisitoire qui en fait une dangereuse charlatanerie scientifique.

La pièce commence (p. 24) par la satisfaction de Ballina, qui trouve concluante sa première expérience de psychanalyse pratiquée sur son patient

15 «Comisario.—¿ Pero cuáles son los locos?

Don PACO. - ¿No los conoce usted por esas voces de energúmenos y esos ademanes descompuestos?

COMISARIO.- ¿Estos...? (Señala a los cuerdos.)

Don PACo.- Sí, hombre, sí... y hay que meterlos en cintura ahora mismo.

Cuerdos. - (Dando grandes voces.) No haga usted caso, señor comisario. Los locos son ésos... Nosotros, somos los cuerdos, sí, señor, los cuerdos.

Don Paco.- Como ve, nosotros somos los locos pacíficos, y ellos los cuerdos de atar.» (Tararí, p. 89-90).

$[\ldots]$

Visitante.- Yo, señor, soy un pobre chiflado. Estoy aquí en medio porque... francamente, ya no sé cuál es mi bando y quisiera que me lo dijese usted.

Comisario.- ¿ ¿Que se lo diga yo? (Mirando a los dos grupos.) Amigo mío, no tengo tanta penetración. (Se queda, durante un momento, pensativo.) Pero, vamos a ver, ¿aquí no había un Director?» (Tararí, p. 92) 
Osuna. Sûr de lui, il s'en vante auprès de son confrère le psychiatre Carrasco: il a pu diagnostiquer la folie de ce «pauvre fou» comme le résultat «d'un choc produit par un sentiment pervers de la sexualité» (p. 24):

CARrasco.- Même en considérant tes observations comme correctes, il faut une grande audace pour s'attaquer d'une forme aussi violente à ce que l'on pourrait appeler un mensonge organisé $[. .$.$] je pense que tu devrais faire attention avec$ ces expériences. Il n'y a rien de plus dangereux que de planter le pieu de la vérité dans un bloc solidement formé par le mensonge, car, en fin de compte, c'est ce que tu fais. ${ }^{16}$

Pointe déjà dans ce dialogue l'ambiguïté, qui ira croissante: la pièce, sous couvert d'examiner ces «mensonges organisés» de la folie et ces techniques psychanalytiques innovantes pour en venir à bout, ne nous parle-t-elle pas avant tout de la dialectique mensonge-vérité, de la question du mensonge tout court et de ses effets quand la vérité vient le défaire? Ne pose-t-elle pas en outre une thèse qui vient contredire l'invention de l'inconscient, tout mensonge impliquant une volonté, et donc une conscience de mensonge comme l'exprime Don Manuel? (Sinrazón, p. 60):

Ballina.- (Avec naturel.) Mais le mensonge a une limite...

D. Manuel.- (Franchement nerveux.) D'accord. Nous voilà d'accord. Mais dites-moi, là, maintenant, en cet instant précis, quelle est la limite du mensonge? Ballina.- Du calme, du calme, don Manuel.

D. MAnuel. - Non, non, non. Le fait est que vous ne pourrez pas répondre, car le mensonge n'a pas de limite, n'a pas de mesure, il ne peut pas avoir de limite. C'est quelque chose en constant mouvement, qui change de place avec nous-mêmes, qui nous est attaché, qui avance, recule, dépend de nos circonstances, de notre énergie, de notre courage et aussi de notre volonté. Oui, oui, docteur, de notre volonté. Il n'est jamais supérieur à nous; il dépend toujours de nous de le situer et donc il est indispensable, là, maintenant, sur le champ, que vous et moi, nous déterminions la limite de notre mensonge...

BALlina.- Mais don Manuel...

D. Manuel.- Oui, oui, oui. Là, maintenant, sur le champ. La limite de notre mensonge est la forclusion de notre palais. Il faut redoubler le contrôle, et que personne n'entre sans notre permission. [...]

Ballina.- Carrasco avait raison. Rien n'est plus dangereux que de planter le pieu de la vérité dans un bloc solidement formé par le mensonge... ${ }^{17}$

16 «Aun dando por ciertas tus observaciones, hace falta una gran audacia para atacar en esa forma tan violenta lo que pudiéramos llamar mentira organizada. [...] creo que tú debes tener cuidado en esos ensayos. No hay más peligroso que meter la verdad como una cuña en un bloque sólidamente formado por la mentira, que, al fin y al cabo, es lo que tú haces.» (p. 25)

17 «Ballina.- (Con naturalidad.) Pero la mentira tiene un límite...

D. Manuel.- (Francamente nervioso.) De acuerdo. Ya estamos de acuerdo. Pero dígame ahora mismo, en este mismo instante, cuál es el límite de la mentira.

BALLinA.- Calma, calma, don Manuel.

D. Manuel. - No, no, no. Si no podrá contestarme, si la mentira no tiene límite, 
Ainsi finit l'acte II. L'acte III révèlera la seconde supercherie de Ballina: il ne mène son expérience de cure par la parole et n'impose la vérité à la fin de la pièce que pour mieux favoriser en réalité son tête-à-tête amoureux avec sa patiente, la reine:

BALLinA.- [...] Lorsque nous sommes seuls, en tête à tête, la reine devient femme, quel plaisir de l'écouter. Si jeune! Si belle! Si sympathique! Si raisonnable!

$[\ldots]$

REINE.- Tous les hommes de mes rêves portaient une couronne.

BALLINA.- Et moi ?18

Ce qui est apparu dans un premier temps comme un débat scientifique sur la meilleure cure pour les patients se révèle n'être qu'un double stratagème psychanalytique et amoureux: provoquer le transfert de la reine certes, mais, ne serait-ce pas surtout se faire réellement aimer d'elle, devenir son roi? Rien n'y fait. Le transfert ne réussit pas et la reine, dans son délire, en aime un autre, celui qu'elle attend, le Roi. À moins qu'elle ne soit plus lucide qu'il n'y paraît et qu'elle se joue du malheureux psychanalyste. Cependant, jusqu'au bout, le doute est maintenu quant aux intentions fluctuantes de Ballina. On croit découvrir la supercherie psychanalytique du médecin quand se révèle sa déconvenue face à une reine qui refuse son amour; mais aussitôt, la pièce réoriente l'interprétation: la déconvenue finalement ne concernerait-elle pas plutôt l'échec du transfert et donc de l'expérience médicale. Le doute aussi affecte la sincérité du docteur quant à l'intérêt réel qu'il porte à ses patients. En effet, tout au long de la pièce s'exprime son mépris le plus absolu pour ses patients qu'il désigne toujours par des expressions comme: «ce pauvre fou» («ese pobre loco», p. 24). Loin de l'objectivité requise, il semble bien être le porteur des préjugés de la société. Don Manuel se bat pour éliminer le mot «fou» - mot interdit dans la fiction

no tiene medida, no puede tener límite. Es una cosa que se mueve continuamente, cambia de sitio con nosotros, va con nosotros mismos, avanza, retrocede, depende de nuestras circunstancias, de nuestra energía, de nuestro valor y también de nuestra voluntad. Sí, sí doctor de nuestra voluntad. No es nunca superior a nosotros; siempre depende de nosotros el situarla y, por lo tanto, es indispensable que ahora mismo, en este mismo instante, tracemos usted y yo el límite de nuestra mentira...

Ballina.- Pero don Manuel...

D. Manuel.- Sí, sí, sí. Ahora mismo, en este mismo instante. El límite de nuestra mentira es la cancela de nuestro palacio. Hay que doblar en ella la guardia, y que no entre nadie sin nuestro permiso. [...]

Ballina.- Tenía razón Carrasco. No hay nada más peligroso que meter la verdad como una cuña en un bloque sólidamente formado por la mentira...» (Sin razón, p. 61-62)

18 «Ballina.- [...] Cuando estamos solos, la Reina se convierte en mujer, da gusto oírla. ¡Tan joven! ¡Tan guapa! ¡Tan simpática! ¡Tan razonable! (Sinrazón, p. 79)

$[\ldots]$

ReINA.- Siempre. Todos los hombres de mis sueños llevaban corona.

Ballina.— ¿Y yo?» (Sinrazón, p. 83). 
du Palais de Béatrice -, manière de nier la réalité des fous; Ballina n’a de cesse de l'employer. Dans Tararí, le Visiteur évite aussi de prononcer le mot «fou», mais pour une raison inverse: prononcer ce mot tabou, c'est donner existence à ce monde, c'est encore risquer la contagion. À la fin de la pièce, le Visiteur, désormais interné parmi les fous, n’a plus peur des mots, il se désigne lui-même comme "un pauvre cinglé» ("un pobre chiflado», Tararí, p. 92).

Le point de départ de Sinrazón est donc une mise en scène de ce qu'est supposée être cette nouvelle technique de la cure par la parole, avec les phénomènes de transfert et de contre-transfert. Ballina expose à plusieurs reprise cette thérapie, avec tous les termes techniques. Il le fait de manière très didactique. Sauf que tout se détraque et la trame psychanalytique se transforme en un classique drame de mœurs avec son triangle amoureux, qui finit mal puisque Don Manuel tue Ballina, devenu son rival amoureux. Les mensonges ainsi révélés laissent planer la plus grande suspicion sur la cure psychanalytique. À la fin de la pièce, nous avons une belle mise en abîme de la technique psychanalytique du divan et du transfert dénoncée comme une manipulation. On y voit comment le docteur Ballina tente d'obtenir de sa patiente... une déclaration d'amour?... le transfert?:

ReIne.- (Avec coquetterie). Tu viendras au bal, docteur? On dansera?

Ballina.- Je viendrai mais maintenant dis-moi (Avec persuasion) Toi, tu es malade. On était d'accord hier sur le fait que tu es malade?

ReIne (Acquiesçant sous le poids de désirs sensuels).- Oui.

Ballina.- Toi, quand tu étais petite, tu avais un portrait du Roi, qui t'a fait tomber amoureuse du monarque.

ReINE.- Tu viendras au bal? On dansera?

Ballina.- Toi, quand tu étais petite, tu es tombée amoureuse d'un portrait du Roi.

ReINe (Hésitante).- Oui.

Ballina.- Toi tu es malade et moi je vais te soigner.

ReINE.- Oui

Ballina.- Bon, raconte-moi maintenant ton dernier rêve. Tu as rêvé de quoi hier soir?

REINE.- Hier soir, hier soir.

Ballina (Il l'allonge sur le divan).- Ferme les yeux et dis-moi de quoi tu as rêvé hier soir. (Don Manuel entre silencieusement et s'apprête à écouter sans être vu.)

ReInE.- Tu viendras au bal? On dansera?

Ballina.- Oui, on dansera mais dis-moi de quoi tu as rêvé. Raconte-moi ton rêve. (Pause. Le médecin s'assied de nouveau.)

REINE.- J'ai rêvé... j'ai vraiment honte.

Ballina.- - Allez. Parle (Il pose sa main sur son front) Parle.

ReINE.- J'ai rêvé de toi... Tu me rendais visite dans ma chambre et j'étais très contente...

BALLINA.- Je portais une couronne?

REINE.- Non. 
BALLina.- Aucun vêtement royal?

REINE.- Aucun.

BALlina.- Quand tu as rêvé d'autres fois à des hommes, ils portaient une couronne?

Reine.- Toujours. Tous les hommes de mes rêves portent une couronne.

Ballina.- Et moi? (Lumière blanche au-dessus de la tête de la Reine.)

Reine.- Non. Tu étais dans mon rêve comme tu es: mince, brun, agréable, sympathique. (Lumière rouge au-dessus de la tête de don Manuel) [...] tes yeux prononçaient des mots que j'entendais pleine de joie... Tu me disais que j'étais malade. Que tu allais me soigner. Que tu allais me sortir d'ici. Que nous serions heureux. Et moi, moi, je te croyais. (Lumière verte au-dessus de la tête du docteur) $)^{19}$.

À première vue, la session analytique paraît normale. Tous les éléments de la cure psychanalytique sont présents: le divan, la cure par la parole, la mise à jours de souvenirs, le récit de rêves, le transfert, le contre-transfert. Cependant, elle est minée de toutes parts. Ballina se tient au plus près de sa patiente, et la

19 «ReIna.- (Con coquetería.) ¿Irás al baile, doctor? ¿Bailaremos?

Ballina.- Iré. Pero ahora dime (Con persuasión.): tú estás enferma. Quedamos ayer en que tú estás enferma.

ReinA.- (Aceptándolo toda tiranizada por sus deseos sensuales.) Sí.

BALlina.- Tú, siendo pequeña, tenías un retrato del Rey, a través de cuyo retrato te enamoraste del monarca.

ReINA.- ¿Irás al baile? ¿Bailaremos?

BALLINA.- Tú, siendo pequeña, te enamoraste de un retrato del Rey.

Reina.- (Vacilante.) Sí.

BALLINA.- Tú estás enferma y yo voy a curarte.

REINA.- Sí

ReInA.- Anoche, anoche.

BAllina.- (La echa en el diván.) Cierra los ojos y dime qué soñaste anoche. (Entra sigilosamente Don Manuel y se dispone a escuchar sin ser visto.)

REINA.- ¿Irás al baile? ¿Bailarás conmigo?

Ballina.- Sí. Bailaremos. Pero dime lo que has soñado. Cuéntame tu sueño.

(Pausa. El médico vuelve a sentarse.)

ReINA.- Soñé... Me da mucha vergüenza.

Ballina.- Vamos. Habla. (Le apoya una mano en la frente.) Habla.

REINA.- Soñé contigo... Me visitabas en mi cuarto y yo estaba muy contenta...

BALLINA. - ¿Llevaba yo puesta una corona?

REINA.- No.

BALLINA. - ¿Ni ninguna prenda del Rey?

ReINA.- Ninguna.

BALLINA. - ¿Cuando has soñado otras veces con hombres, no llevaban corona?

Reina.- Siempre. Todos los hombres de mis sueños llevaban corona.

BALlina.- ¿Y yo? (Sobre la figura de la Reina una luz blanca.)

Reina.- No. Tú eras en mi sueño como eres: delgado, agradable, simpático. (Sobre la cabeza de don manolito una luz roja.) Y tus ojos [...] pronunciaban palabras que yo oía llena de regocijo... Me decías que yo estaba enferma. Que tú ibas a curarme. Que me ibas a sacar de aquí. Que seríamos felices. Y yo, yo te creía. (Sobre la cabeza del doctor una luz verde.)»(Sinrazón p. 81-83). 
touche même: assis sur le divan où elle est allongée, il pose sa main sur son front. Les questions et suggestions de Ballina orientent clairement les réponses de la patiente, et Ballina les interprète selon ce qu'il souhaite entendre. Sans parler de la présence d'un observateur, don Manuel, qui interprète la scène de transfert-contre-transfert comme une scène de séduction. N'est-ce pas ce qu'elle est ici en réalité? La psychanalyse est ainsi disqualifiée et présentée comme une escroquerie destinée à servir des fins amoureuses inavouables.

Dans Tararí, avec plus de force encore que dans Sinrazón, la toute-puissance de la parole médicale impose sa vérité au patient. Loin de mettre la parole au service de la cure, l'analyste impose ses vues, n'hésitant pas à manipuler et à mentir. Ce que signale le fou, don Paco, qui a pris la place du Directeur et accueille le riche commerçant venu se renseigner pour faire interner son frère: «Lorsque on entre dans cette maison on ne vous dit jamais la vérité.» (p. 39). Si dans Sinrazón la reine résiste à son analyste, le Visiteur dans Tararí est prompt à accepter ce que Don Paco, dans son double rôle de psychanalyste et directeur de l'asile, lui impose. Don Paco met en œuvre ce qu'il a appris des techniques des psychiatres-psychanalystes, et s'applique à son tour à rendre fou celui qui ne l'est pas, en l'occurrence le pauvre visiteur. En copie conforme de l'analyste, il l'invite à libérer sa parole, à raconter sa vie, exploite la moindre faille, l'embrouille. On le voit passé maître dans l'art de la manipulation. Il réussit ainsi à convaincre le pauvre visiteur que non seulement il n'est pas ce qu'il est, mais en plus qu'il y a tout avantage. Morceau de bravoure de la caricature de la maïeutique freudienne à l'œuvre dans cette pièce: "Cela ne date pas d'aujourd'hui. Ça vient de plus loin ${ }^{20}$.", diagnostique-t-il, avant d'inviter le malheureux à exposer ses traumatismes passés: «Dites, dites tout ce que vous voulez; épanchez-vous, et vous vous sentirez beaucoup plus tranquille ${ }^{21}$.» La critique de la psychanalyse est d'autant plus acerbe que la méthode de l'analyste est menée ici par un imitateur dans un contexte de supercherie, mettant ainsi en évidence ce qu'il y a à penser de la cure par la parole. Nous voyons comment la prétention de la cure freudienne à faire émerger la vérité du patient se résout dans la construction d'une fiction dirigée: le commerçant n'est pas ce qu'il croit être, il vient demander son internement. La supposée neutralité du psychanalyste est mise à mal puisque ce qui se produit est un tour de passe-passe manipulatoire, qui, lorsque c'est nécessaire, est appuyé par une menace à peine voilée: «Don Paco.— Mon Dieu, calmez-vous... calmez-vous. Pour rien au monde je ne voudrais que vous vous énerviez. Ne soyez pas si nerveux, parce que sinon ce serait horrible et nous serions obligés de... non, non, ce serait très désagréable ${ }^{22}$.»

20 «No es de hoy. Eso tiene que venir de atrás.» (Tararí, p. 41).

21 «Don Paco.- Diga, diga usted lo que quiera; desahóguese, así quedará usted mucho más tranquilo.» (Tararí, p. 41).

22 «Don Paco.- Por Dios, tranquilícese... tranquilícese. Por nada de este mundo quisiera que se alterara usted. No se ponga nervioso, porque entonces sería horrible y nos obligaría a... no, no, sería muy desagradable.» (Tararí, p. 40). 


\section{«Rien de plus dangereux que de planter le pieu de la vérité dans un bloc solidement formé par le mensonge»}

Cependant, dans Tararí - comme c'est le cas aussi de Sinrazón - la critique de la psychanalyse s'arrête là, dans ces jeux comiques et situations cocasses, où peuvent s'enchaîner et se déchaîner les jeux de mots, les malentendus, les quiproquos, c'est-à-dire toutes ces situations qui plaisent tant au spectateur des comédies légères de l'époque. La critique porte surtout sur la société espagnole et ses lieux d'internement et d'exclusion. Qui l'asile enferme-t-il du commerçant ou du philosophe? Assurément ce dernier. Dans le monde à l'envers que construit la révolte des fous, en appliquant cette logique bourgeoise, c'est bien encore le philosophe qui est interné comme fou, sauf que la folie subversive de ces fous, qui ont beaucoup appris des techniques répressives et manipulatoires de ceux qui les ont internés, s'arrange pour que soit interné justement le commerçant et non plus le philosophe. Véritable justice théâtrale à la fin de ce premier acte. La séquence aura illustré comment: «Dans la réalité, l'enjeu n'est pas une arme mais une étiquette: celui qui réussit le premier à la poser sort vainqueur de la bataille; l'autre étiqueté est réduit au rôle de victime.» (Jaccard 1992:35). Tout au long de la pièce d'Andrés Álvarez, l'argument suprême qui revient est: «j'imagine que si on vous a interné, ce n'est pas pour rien.»

VISITEUR.- J'imagine que si on vous a interné, ce n'est pas pour rien.

L'administrateur. - Eh bien non, Monsieur. On m’a enfermé, oui. Et alors? Je suis toujours celui que j'étais; je me sens comme je me sentais et comme je l'ai toujours été.

Visiteur.- Oui, c'est ça; chez vous c'est de naissance. ${ }^{23}$

Dans le deuxième acte de Tararí, nous assistons à l'assemblée des fous qui ont pris le pouvoir, donc des «nouveaux maîtres qui [vont] imposer leur raison à tous les hommes»(Tararí, p. 49). Chacun y va de ses propositions pour apporter le bonheur aux hommes et changer la société. Les échanges mêlent des considérations sociales et des considérations de type théologique tirées de la morale chrétienne: l'un des fous propose que tous les hommes soient millionnaires. Le Fou 1 manifeste alors son désir d'être le seul pauvre parmi tous ces millionnaires. "Je ne vois pas l'avantage d'être pauvre», l'interpelle le Fou 3. Reproduisant l'argument du Pauvre dans le Grand théâtre du monde de Calderón de la Barca, le Fou 1 rappelle alors l'une des plus grandes supercheries pour justifier les inégalités sociales, et même leur nécessité, et garantir ainsi l'ordre social dans la très catholique monarchie de l'Espagne du XVII ${ }^{\mathrm{e}}$ siècle: celle du rôle de la charité: «Imagine-toi, que si je suis le seul pauvre au monde, tous les hommes devront compter avec moi pour être bons et charitables. Eh

23 «Visitante.- Me figuro que si lo tienen encerrado será por algo.

Administrador.- Pues no, señor. Me han encerrado, sí. ¿Y qué? Yo soy el mismo que era; estoy como estaba, como he estado siempre.

Visitante.- Vamos, sí; lo de usted es de nacimiento.» (Tararí, p. 57) 
bien, ce n'est pas rien d'être la seule personne sur terre à qui il faudrait faire des faveurs pour gagner le ciel.» (Tararí, p. 52). Dans l'Espagne des années 1920, où la question de la justice sociale et le poids de l'église sont au premier plan des débats et fractionnent la société, le message ne peut qu'être entendu.

Dans Sinrazón, la première image des fous que nous avons est celle d'une horde en haillons: "(Des fous arrivent précipitamment. On dirait des vagabonds, portant des vêtements sales et des haillons. ${ }^{24}$ ». L'Église est largement représentée, les sœurs du couvent de Sainte Ursule, le fou qui se prend pour un évêque, et la reine très catholique.

Deux fous apparaissent dotés de la clairvoyance qu'on attribue aux bouffons et aux fous: Don Paco dans Tararí et Don Manuel dans Sinrazón. Ils sont les voix subversives, lucides et démystificatrices qui dénoncent une société excluante, minée par les préjugés, qui a inventé des espaces d'enfermement pour ceux qu'elle a catégorisés comme déviants. Don Paco, jetant son regard surplombant, distancié et ironique, prend des allures d'anarchiste visionnaire:

Don PAco.- Non, non pas d'extrémismes. Mon programme est le suivant: qu'il n'y ait pas plus de riches que les riches, ni plus de pauvres que les pauvres. J'obligerai tous les malades à être malades, toutes les femmes à être femmes et tous les hommes à être hommes. J'obligerai les travailleurs à travailler et les paresseux à ne rien faire. Ah! Et j'obligerai les malades en phase terminale à mourir.

Fou 2.- En voilà une affaire! Mais c'est là laisser les choses comme elles sont.

Don Paco._- Oui, mon ami, oui. Mais pour laisser les choses comme elles sont, il faut faire une sacrée révolution!25

Et la psychanalyse, cette science des profondeurs, capable de faire parler l'inconscient, que peut-elle face à une société en décomposition, où les inégalités sociales augmentent, où les riches sont chaque fois plus riches et les pauvres chaque fois plus pauvres, où chacun est sommé de ne pas être ce qu'il est pour le bon ordre de la société? Que peut la psychanalyse, produit de la bourgeoisie et pour la bourgeoisie, pour ces «hordes de fous en haillons» du "palais de la reine Béatrice»? Ne participe-t-elle pas du grand mensonge social? Don Paco énonce sa conclusion finale dans la dernière réplique de Tararí: "Calmez-vous, calmez-vous, et écoutez mon conseil: cela vous rapportera plus de faire partie des pacifiques, car sinon on vous mettra une camisole de force.». La vérité est subversion qui mène à la camisole de force. «Il n'y a rien de plus dangereux que

24 «(Van llegando locos atropelladamente. Visten como vagabundos, con ropa sucia y harapienta)» (Sinrazón, p. 34)

25 «Don PAco. - Nada, nada de extremismos. Mi programa es que no haya más ricos que los ricos, ni más pobres que los pobres. Obligaré a todos los enfermos a estar enfermos, a todas las mujeres a ser mujeres y a todos los hombres a ser hombres. Obligaré a trabajar a los trabajadores y a no hacer nada a los holgazanes. ¡Ah! Y obligaré a morirse a los enfermos de la última.

Loco $2^{\circ}$.- ¡Vaya una cosa! Pero si eso es dejar las cosas como están.

Don Paco.- - Sí, amigo, sí. Pero para dejar las cosas como están ¡menuda revolución hay que hacer!» (Tararí, p. 52-53) 
de planter le pieu de la vérité dans un bloc solidement formé par le mensonge.», répète plusieurs fois le docteur Ballina dans Sinrazón. La vérité conduit au chaos. Ce sont là les deux conclusions désabusées et pessimistes que proposent ces farces, comiques ou tragiques, selon l'angle d'où on les lit et on les regarde.

\section{Bibliographie}

Andrés Álvarez V., 1929, Tararí, farsa cómica en dos actos y un epílogo, Madrid, Revista de Occidente, Nova Novorum.

Calderón de la Barca, 1717, La cena de Baltasar, dans Autos sacramentales alegóricos e historiales del insigne poeta español Pedro calderón de la Barca, Obras posthumas, Primera parte, Madrid, Imprenta Manuel Ruiz.

Derrida J., 2005, Histoire du mensonge, Prolégomènes, Paris, l'Herne.

Freud S., (1943) 2006a, Métapsychologie, Paris, Gallimard, «folioessais».

Freud S., (1942) 2006b, Sur le rêve, Paris, Gallimard, «folioessais».

Grimaldi N., 2011, «La Folie», dans La Folie, Paris, Fayard.

Grimaldi N., 2009, Une démence ordinaire, Paris, PUF.

Heers J., 1983, Fête des fous et carnavals, Paris, Fayard.

Jaccard R., 1992, La Folie, Paris, PUF.

Sánchez Mejías I., 2010, Sinrazón: juguete trágico en tres actos, Sevilla, Espuela de Plata, col. «El Teatro Moderno». 